\title{
Dual Pathology- Clear Cell Variant of Renal Cell Carcinoma and High Grade Papillary Urothelial Carcinoma of Urinary Bladder a Rare Entity: Case Report
}

\author{
Mukherjee Rina ${ }^{1}$,Ganguly Tanmoy ${ }^{1}$, Bandopadhyay Jayeeta ${ }^{2}$,Das Dipkana ${ }^{3}$, \\ Singh Neha ${ }^{4}$ \\ ${ }^{1}$ Senior Consultant\&HOD, ${ }^{1,2,3}$ Senior Resident, ${ }^{4}$ DNB Post Graduate Trainee \\ Department Of Pathology, B. R. Singh Hospital AndCentre For Medical Education \&Research, Eastern \\ Railway, Sealdah, Kolkata-700014, India
}

\begin{abstract}
Simultaneous occurrence of clear cell variant of renal cell carcinoma and high grade papillary urothelial carcinoma of urinary bladder is a rare entity. The review of different literatures revealed incidence of carcinoma of urinary bladder and the prostate in patients with renal cell carcinoma, especially the papillary subtypes.Here we report a case of 61 years male patient presented with hematuria of one month duration. Further investigations revealed SOL in upper pole of left kidney measuring $4 \times 4 \times 3.5 \mathrm{~cm}$ and a separate friable growth in theurinary bladder. Urinary Bladder tumorspecimen was diagnosed as high grade papillary urothelial carcinoma of urinary bladder and left radical nephrectomy specimen was diagnosed as Clear cell variant of renal cell carcinoma. The etiopathogenesis of this association remains debated. It seems to be based on genetic alterations that have not been formerly proved.
\end{abstract}

Keywords: Clear cell variant of renal cell carcinoma, high grade papillary urothelial carcinoma of urinary bladder, SOL in upper pole of left kidney, Radical nephrectomy.

\section{Introduction}

Several studies in the literature dealing with the occurrence of multiple primary malignant neoplasms report incidence rates ranging from 2.6 to $3.9 \%$ among cancer patients ${ }^{1,2}$ and those studies that include tumors found at autopsy report even higher rates ${ }^{3}$. The most common sites for multiple tumors vary among the reports but as the occurrence of primary tumors in the genitourinary (GU) tract is higher compared to other organ systems, multiple tumors have a predilection to involve a GU tract organ.Multiple primary malignant tumors involving only the GU tract are rare and present either as multicentric malignant tumors of the urinary epithelium, tumors occurring in bilateral organs or as multiple tumors with dissimilar histology.We report a 61 years old male patient who first underwenttransurethral resection of bladder tumor(TURBT) and reported as high grade papillary urothelial carcinoma of urinary bladder with squamoid differentiation. Later he underwent left radical nephrectomy and found to have clear cell variant of renal cell carcinoma.

\section{Case Report}

A 61 years old gentleman, known smoker,presented with complaints of burning sensation during micturition, abdominal pain and heaviness along with few episodes of hematuria and weakness of one month duration.On clinical examination, masswasfelt on the left flank.Investigation includesradio-imaging studies like USG KUB, MDCT Urography and PET CT,showed a large solid-cystic lesion in upper pole of left kidney measuring $5.5 \times 5.1 \times 6.3 \mathrm{~cm}$ and a separate neoplastic lesion in urinary bladder involving left lateral \& posterior wall extending to the base measuring $7.1 \times 5.1 \times 5.5 \mathrm{~cm}$.Cystoscopy showed a vascular and friable tumor involving base, left lateral wall and anterior wall around bladder neck. Transurethral resection of bladder tumor was done and sent for histopathological examination. It was reported as high grade papillary urothelial carcinoma of urinary bladder with squamoid differentiation, invading the muscularis layer.

For kidney tumor, left radical nephrectomy was done and sent for histopathological examination. On cut section, a solid, well circumscribed variegated yellowish tumor in upper pole of the kidney measuring $4 \times 4 \times 3.5 \mathrm{~cm}$, sharply demarcated from the surrounding tissues with foci of haemorrhage, necrosis and cystic changes was noted. Renal pelvis,ureter, renal vessels, and pericapsular area were grossly unremarkable. Histopathological examination revealed a pT1aNxMx, clear cell variant of renal cell carcinoma, Fuhrman grade II.No pericapsular extension was identified. Ureter and renal vessels were free of tumor invasion. No lymph node was found.

Neo-adjuvant chemotherapy followed by radical cystectomy is awaited. 


\section{Images:}

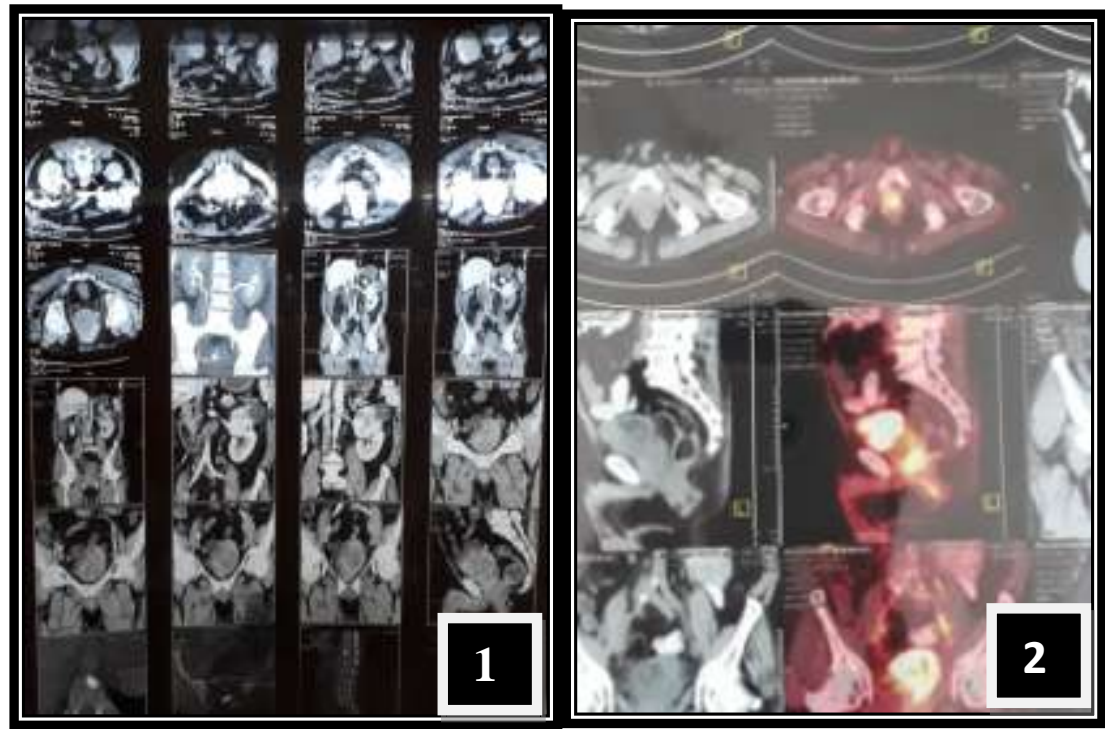

Fig 1\&2: MDCT Urography and PET CT image showing left kidney and urinary bladder tumor.

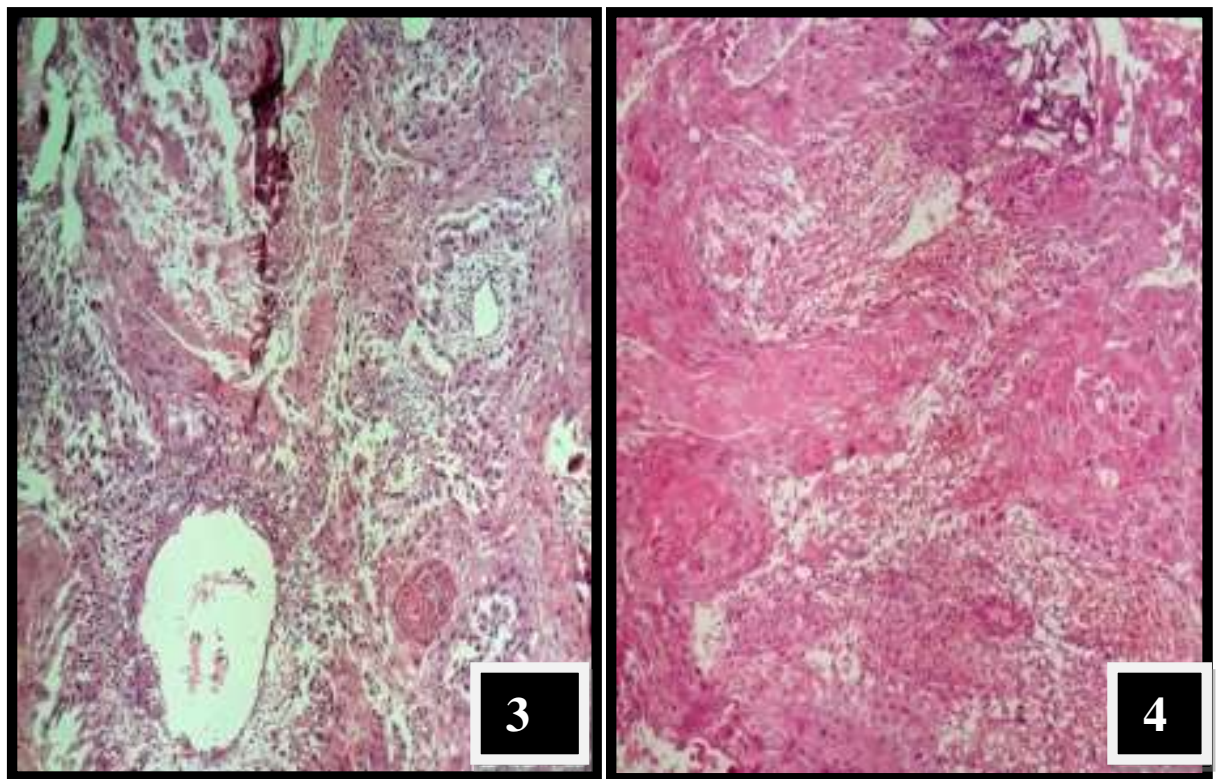

Fig 3 \& 4:Photomicrographs of TURBT specimen showing high grade papillary urothelial carcinoma of urinary bladder with squamoid differentiation. 


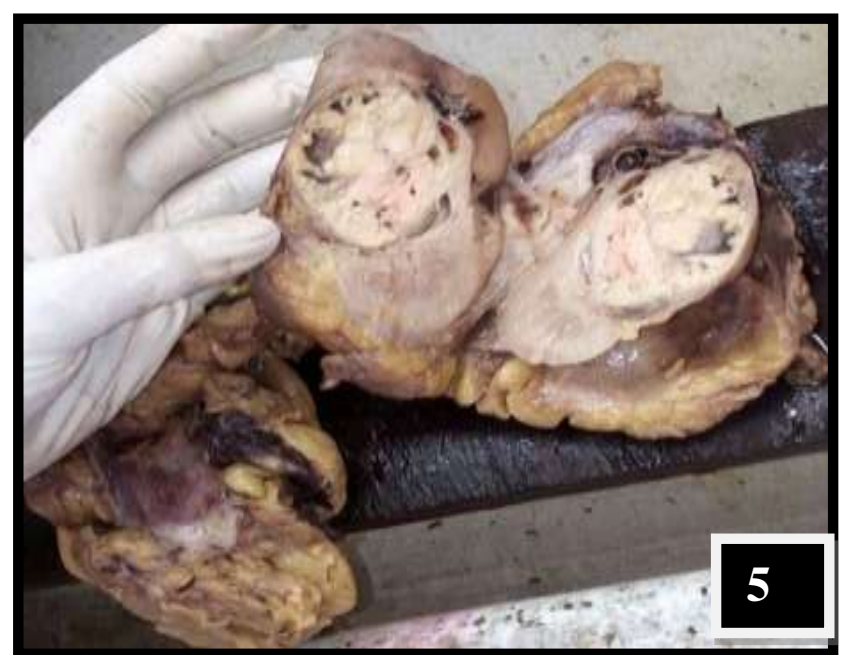

Fig 5: Gross image of well circumscribed left renal tumor in upper pole.

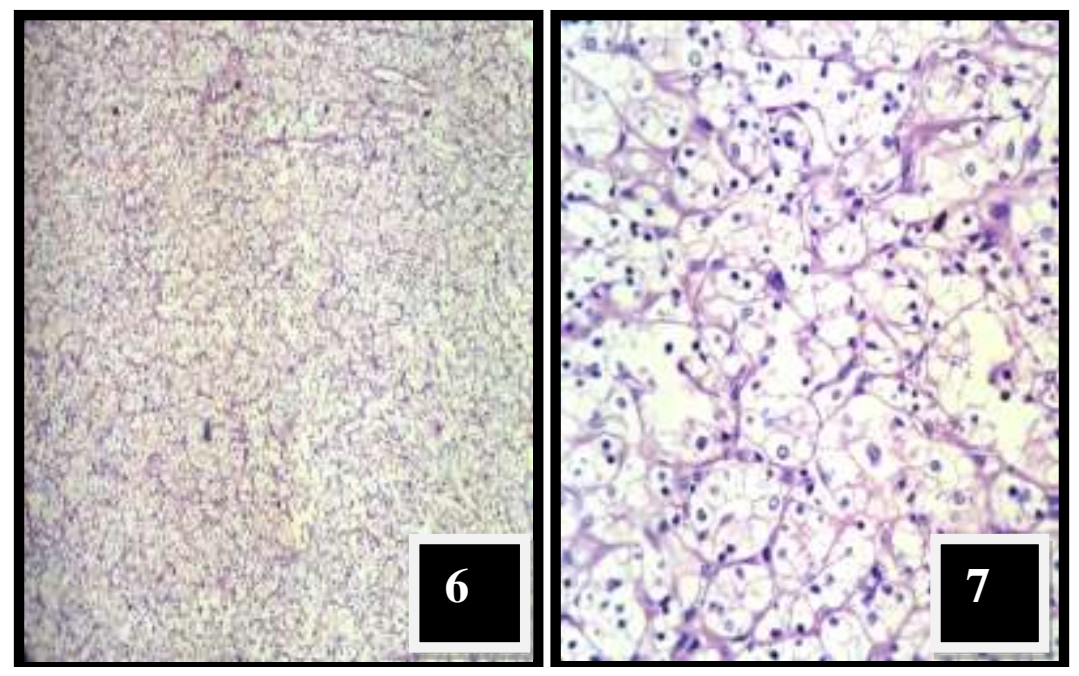

Fig 6 \& 7: Photomicrograph of clear cell variant of renal cell carcinoma (Left kidney)

\section{Discussion}

Clear cell variant is the most common subtype of renal cell carcinomaand papillaryurothelial carcinoma isthe most common urinary bladder carcinoma. Multiple primary malignant tumors can occur in all decades, but they are most common in the geriatric population. It is a well known fact that urinary tract is one of the most common sites for multiple primary tumors. Extensive search revealed that multiple primary tumors occur in $3.1 \%$ of total cancer patients and $13.9 \%$ of these have genitourinary tumors ${ }^{4}$.Multiple malignant tumors involving more than one genitourinary site are rare.In one study, 5.4\% of the patients with urinary bladder neoplasms had malignant tumors outside the urothelium and of these $4.3 \%$ were renal cell carcinomas ${ }^{5}$. Several studies done by Begget $\mathrm{al}^{6}$, Neuzillet et $\mathrm{al}^{7}$, Jensen et $\mathrm{al}^{8}$ and others ${ }^{9}, 10,11$ concluded that urinary bladder cancer patients have higher risk of development of renal cell carcinoma.Neuzilletet $\mathrm{al}^{7}$ and Rabbaniet $\mathrm{al}^{9}$ concluded that this dual association is usually observed in papillary subtypes of renal cell carcinoma.But in our study, this dual association was observed between the clear cell variant of renal cell carcinoma and high grade papillary urothelial carcinoma of urinary bladder. Extensive search does not reveal such coexistence in published literature.Smoking, drinking, alcohol,oncogenic substances (like - radiation) and genetic mutations are the common risk factors for the development of primary cancers like renal and urothelial carcinomas ${ }^{11,12}$.

Some studies reported that the chemo and/or radio therapy for one cancer may induce the secondary carcinoma ${ }^{13}, 14$. But in our study no such intervention was done. The carcinogenesis is an anomalous phenomenon determined by a clonal selection. Association of dual primary cancers affecting different organs necessitates the coexistence of genetic mutationsor a multifactorial etiology. 


\section{Conclusion}

This case reported as a coexistence of clear cell variant of renal cell carcinoma with high grade papillary urothelial carcinoma of urinary bladder in an adult smoker. This dual association is least reported in different literature. Due to our limited resources we could not establish whether this association is due to some genetic alterations or it is just an incidental finding.But further researches are necessary to establish the actual etiopathogenesis of such togetherness.

\section{References}

[1]. Okamoto N, MorioS, Inoue R, Akiyama K. The risk of a second primary cancer occurring in five year survivor of an initial cancer. Jpn j ClinOncol. 1987; 17:205-13.

[2]. Storm HH, Jensen OM, EwertzM, LyngeE,Olsen JH, SchouG, OsterlindA. Summary: multiple primary cancer in Denmark, 194380. Nattl Cancer Inst Monogr. 1985; 68:411-30.

[3]. Suen KC, Lau LL, Yermakov V. Cancer and old age: an autopsy study of 3533 patients over 65 years old. Cancer 1974:33:11641168 .

[4]. Matzkin M, Braf Z. Multiple primary malignant neoplasms in the genitourinary tract: occurrence and etiology. J Urol 1989;142:112.

[5]. Ward-McQuaid JM. Carcinoma of the bladder and multiple unrelated malignancy. Brit J Urol 1963:35:169-174.

[6]. BeggCB, Zhang ZF, Sun M, Herr HW,SchantzSP. Methodology for evaluating the incidence of second primary cancer with application to smoking-related cancers from surveillance, Epidemiology, and End Result (SEER) program. Am J Epidemiol 1995;142:653-65.

[7]. Neuzillet Y, Lechevalier E, Coulange C. Cancer du rein et deuxièmecancer :Analyse critique de la littérature. Prog en urol 2007 17:35-40.

[8]. Jensen OM, Knudsen JB, Sorensen BL. Second cancer following cancer of the urinary system in Denmark; 1943-80. Natl Cancer Inst Monogr 1985;68:349-60

[9]. Rabbani F, Reuter VE, Katz J, Russo P. Second primary malignancy associated with renal cell carcinoma: influence of histologic type. Urology 2000;56:399-403.

[10]. Czene K, Hemminki K. Familial papillary renal cell tumors and subsequent cancers: a nationwide epidemiological study from Sweden. J Urol 2003;169:1271-5.

[11]. Beisland C, Talleraas O, Bakke A Norstein J. Multiple primary malignancies in patients with renal cell carcinoma : a national population-based cohort study. BJU Int2006;97:698-702.

[12]. Sharpe CR, Siemiatycki J, Parent ME. Activities and exposures during leisure and prostate cancer risk. Cancer Epidemiol Biomarkers Prev. $2001 ; 10: 855-60$.

[13]. Travis LB, Rabkin CS, Brown LM, AllanJM,Alter BP, Ambrosone CB, Capoaso N, Chanock S, Demichele A, Figg WD, Gospodarowicz MK, Hall EJ, Hisada M, InskipP, Kleinerman R, Little JB, Malkin D, Ng AK, Offit K, Pui CH, Robinson LL, Rothman N, Shields PG, Strong L, Taniguchi T, Tucker MA, Greene MH. Cancer survivorship-genetic susceptibility and second primary cancers: research strategies and recommendations. J Natl Cancer Inst 2006:98:15-25.

[14]. Smith MT, Skibola CF, Allan JM, Morgan GJ. Causal models of leukaemia and lymphoma. IARC Sci Publ. 2004 ;157:373-92. 